\title{
ON ORDERS AND TYPES OF AN ENTIRE FUNCTION OVER $\mathscr{C}^{k}$
}

\author{
J. GOPALA KRISHNA and I. H. NAGARAJA RAO
}

(Received 16 September 1969, revised 23 July 1970)

Communicated by E. Strzelecki

The concepts of order and type associated with an entire function over the complex plane admit different "natural" extensions, most of which are in vogue, for the case of an entire function over $\mathscr{C}^{k}$, the cartesian product of $k$ copies of the complex plane. This work is concerned with the relations among such extended concepts and with the analogous properties of the concepts.

Throughout we write $f$ to denote an entire function over $\mathscr{C}^{k}$ and $\mathscr{M}=\mathscr{M}_{f}$ to denote its maximum modulus defined at each point $r$ of the set by

$$
\left|\mathscr{C}^{k}\right|=\left[r: r=\left(r_{1}, \cdots, r_{k}\right) \text {, where each } r_{j} \text { is real and } \geqq 0\right]
$$

$$
\mathscr{M}(r)=\max \left[|f(z)|: z \in \mathscr{C}^{k},|z|=\left(\left|z_{1}\right|, \cdots,\left|z_{k}\right|\right)=r\right] .
$$

Fuks and Ronkin (see $\$ 26.2$ of [2], cf. [5]) introduced the concepts of orders and types of $f$ based on an asymptotic comparison, as

$$
\|r\|=\sum_{1 \leqq j \leqq k} r_{j} \rightarrow+\propto \quad \text { (in }\left|\mathscr{C}^{k}\right| \text { ), }
$$

of $1 \mathrm{n}^{+} \mathscr{M}(r)$ with $\sum_{1 \leqq j \leqq k} T_{j} r_{j}^{\alpha j}$ corresponding to different positive real numbers $T_{j}, \alpha_{j}(1 \leqq j \leqq k)$. One might consider $r \rightarrow+\bar{\propto}=(+\propto, \cdots,+\propto)$ instead of $\|r\| \rightarrow+\propto$. In $\S 3$ we show that (see (3.1)) such a consideration gives rise to no new concepts and indicate how advantage may be taken of this fact in discussing the properties of the Fuks-Ronkin concepts.

Another natural approach is to compare $\ln ^{+} \mathscr{M}(r)$ with

$$
\operatorname{Tr}^{\alpha}=\operatorname{Tr}_{1}^{\alpha_{1}} r_{2}^{\alpha_{2}} \cdots r_{k}^{\alpha_{k}}
$$

as $r \rightarrow+\infty$, for different positive real numbers $T, \alpha_{j}(1 \leqq j \leqq k)$ (see [3], [4]; cf. [1] with the help of $\S 5$ ). We attempt a systematic study of the properties of the concepts, the product orders and the product types of $f$, arising out of such a comparison, in $\S 2$. The product orders (of $f$ ), in particular, adimt properties more elegant although less easily established than their analogues for Fuks orders (see (2.1)). 
In $\$ 4$ we consider the concepts of orders and types of $f$, based on special exhaustions of $\mathscr{C}^{k}$, due to Gol'dberg. It turns out that the nondependence of Gol'dberg order on the "fundamental domain" is only a property of $\ln ^{+} \mathscr{M}$ as a real valued "increasing" (see $\S 1$ ) function with no further reference to $f$. In $\S \S 3$ and 4 we attempt to characterise the relations (see (3.7), (4.3)) among the different concepts of orders of $f$. The relations, in particular, show that the property that $f$ is of "finite order" (see $\S 1$ ) is independent of the particular order-concept among the ones under consideration. We however have certain interesting questions, which we indicate at the appropriate places, for which this paper has no answers (in particular see remark (4.6)).

\section{Notation}

We freely make use of the standard notation and conventions of [2] and the ones mentioned earlier. We refer to $\left(\alpha_{1}, \cdots, \alpha_{k}\right),\left(n_{1}, \cdots, n_{k}\right),\left(z_{1}, \cdots, z_{k}\right)$, etc. $\in \mathscr{C}^{k}$ by their respective unsuffixed symbols; $\alpha, n, z$, etc. We take the Taylor expansion of $f$ about $\overline{0}=(0, \cdots, 0) \in \mathscr{C}^{k}$ to be specified by

$$
f(z)=\sum_{n \in I} a_{n} z^{n}, \quad \text { for } z \in \mathscr{C}^{k},
$$

where $I=I^{k}=\left[n: n \in\left|\mathscr{C}^{k}\right|\right.$, where each $n_{j}$ is a rational integer $]$.

We throughout regard $\left|\mathscr{C}^{k}\right|$ as a subspace of a Euclidean space and write int $E$ for the interior of any $E \subseteq\left|\mathscr{C}^{k}\right|$. Corresponding to $r, s \in\left|\mathscr{C}^{k}\right|$, we write

$$
r^{s}=r_{1}^{s_{1}} r_{2}^{s_{2}} \cdots r_{k}^{s_{k}}
$$

where $r_{j}^{s j}$ should be taken as 1 if $r_{j}=s_{j}=0$ and we say that $r \leqq s$ or $s \geqq r$ iff (if and only if) $r_{j} \leqq s_{j}$ for $1 \leqq j \leqq k$, that $r<s$ or $s>r$ iff $r \leqq s$ but not $=s$ and that $r \ll s$ or $s \gg r$ iff $r_{j}<s_{j}$ for $1 \leqq j \leqq k$. We write

$$
\mathscr{C}^{k+}=\left[r: r \in\left|\mathscr{C}^{k}\right| \text { and } \gg \overline{0}\right]
$$

and $r / s=\max \left(r_{j} / s_{j}: 1 \leqq j \leqq k\right)$, when $r \in\left|\mathscr{C}^{k}\right|$ and $s \in \mathscr{C}^{k+}$. We say that a real valued function $\phi$ with domain $E \subseteq\left|\mathscr{C}^{k}\right|$ is increasing, iff $\phi(r) \leqq \phi(s)$ whenever $r, s \in E$ and $r \leqq s$.

Let $\phi$ be a nonnegative real valued function over $\left|\mathscr{C}^{k}\right|$. The closure $P$ of the set (possibly empty),

$$
\left[\alpha: \alpha \in \mathscr{C}^{k+}, \phi(r) \leqq r^{\alpha} \text { as } r \rightarrow+\infty\right]
$$

will be referred to as the product order set of $\phi$ and only a boundary point of $P$ will be referred to as a product order of $\phi$. We say that $\phi$ is of infinite or finite product order according as $P$ is empty or nonempty. Corresponding to a product order $\rho \in \mathscr{C}^{k+}$ of $\phi$, we define the product type $\tau$ (of $\phi$ ) as $+\infty$ or

$$
\inf \left[T: T \in \mathscr{C}^{1+}, \phi(r) \leqq T r^{\rho} \text { as } r \rightarrow+\bar{\infty}\right]
$$


according as the set under consideration is empty or nonempty. By the sum order set $S$ of $\phi$ we mean the closure of the set

$$
\left[\alpha: \alpha \in \mathscr{C}^{k+}, \phi(r) \leqq \sum r_{j}^{\alpha j} \text { as } r \rightarrow+\infty, \text { where } \Sigma=\sum_{1 \leqq j \leqq k}\right]
$$

and by a sum order of $\phi$ we only mean a boundary point of $S$. As in the case of product orders we say that $\phi$ is of infinite or finite sum order according as $S$ is empty or nonempty. By a sum type corresponding to a sum order $\rho \in \mathscr{C}^{k+}$ of $\phi$, we only mean a boundary point of the sum type set corresponding to $\rho$, viz, the closure of

$$
\left[t: t \in \mathscr{C}^{k+}, \phi(r) \leqq \sum t_{j} r_{j}^{p j} \text { as } r \rightarrow+\bar{\infty}\right] .
$$

By a Fuks order of $\phi$ we only mean a boundary point of the Fuks order set of $\phi$ viz, the closure of

$$
\left[\alpha: \alpha \in \mathscr{C}^{k+}, \phi(r)<\sum r_{j}^{\alpha j} \text { as }\|r\| \rightarrow+\infty\right]
$$

(note " $<$ " and " $\|r\| \rightarrow+\infty$ ", see remark (3.12)). By a Ronkin type corresponding to a Fuks order $\rho \in \mathscr{C}^{k+}$ of $\phi$ we only mean a boundary point of the Ronkin type set corresponding to $\rho$ viz, the closure of the set

$$
\left[t: t \in \mathscr{C}^{k+}, \phi(r) \leqq \sum t_{j} r_{j}^{\rho j} \text { as }\|r\| \rightarrow+\infty\right]
$$

We postpone the introduction of the less similar concepts due to Gol'dberg until they become relevant (see $\$ 4$ ).

As usual we attribute all the concepts of orders, types and the related ones associated with $\ln ^{+} \mathscr{M}$ to $f$ itself.

\section{Product orders and types}

Throughout this section we write $\psi$ to stand for a real valued function on $I$ with the property that the limsup and the liminf of $\psi(n) /\|n\|$, as $\|n\| \rightarrow+\infty$, are both finite and positive, so that in particular, $\psi(n)$ could be $\|n\|$ or $n / \alpha$ $=\max \left(n_{j} / \alpha_{j}: 1 \leqq j \leqq k\right)$ for $n \in I$, where $\alpha \in \mathscr{C}^{k+}$ and is independent of $n$. The simpler nature of the product order set $P(f)$ of $f$ over that of its Fuks order set is brought out by the less easily proved (cf. $\$ 26.2$ of [2])

THEOREM 2.1. Let the product order set $P(f)$ of $f$ be nonempty. Then it is an "infinite rectangle with a unique minimal element $\rho$ " i.e. there exists a $\rho \in\left|\mathscr{C}^{k}\right|$ such that

$$
P(f)=\left[\alpha: \alpha \in\left|\mathscr{C}^{k}\right| \text { and } \geqq \rho\right] .
$$

Further this minimal element $\rho$ of $P(f)$ is given (under certain obvious conventions followed in [2]) by

$$
\rho_{j}=\limsup _{\|n\| \rightarrow+\infty}\left\{n_{j} \ln \psi(n)\right\} /\left(-\ln \left|a_{n}\right|\right), \text { for } 1 \leqq j \leqq k .
$$


It is convenient, for later use as well, to distinguish the different stages of the proof as lemmas. We encounter an intricacy characteristic of the discussions in the case of the product concepts in the crucial

LEMMA 2.2. (a) Let $T \in \mathscr{C}^{1+}$ and $R, \alpha \in \mathscr{C}^{k+}$ and $n \in\left|\mathscr{C}^{k}\right|$ but $>\overline{0}$. Let $g$ be the function defined by

$$
g(r)=\left(1 / r^{n}\right) \exp \left(T r^{\alpha}\right), \text { for } r \in\left|\mathscr{C}^{k}\right|
$$

Then $\inf _{r \geqq R} g(r)=\min _{1 \leqq j \leqq k}\left[\inf \left\{g(r): r_{j} ; \geqq R_{j}, r_{m}=R_{m}\right.\right.$ for $\left.\left.1 \leqq m \leqq k, m \neq j\right\}\right]$.

(b) Let further $n / \alpha>T R^{\alpha}$. Then $\inf _{r \geqq R} g(r)=\left[e T R^{\alpha} /(n / \alpha)\right]^{n / \alpha} / R^{n}$.

ProOF of 2.2. We prove (a) by induction on $k$. (a) is trivial if $k=1$. We shall assume (a) with $k-1$ in the place of $k$. Now the technique essentially consists of considering the infimum of $g$ over the different "rays from $\overline{0}$ into $\mathscr{C}^{k+}$ ", Let $a \in \mathscr{C}^{k+}$. It is easily seen that the function $h$ defined by $h(t)=g(t a)$, for $t \in \mathscr{C}^{1+}$, is decreasing in $\left(0, t^{\prime}\right]$ and increasing in $\left[t^{\prime},+\infty\right)$, where the point $r=t^{\prime} a$ satisfies: $T r^{\alpha}=\|n\| /\|\alpha\|$. By considering different $a \in \mathscr{C}^{k+}$ we get that

$$
\inf _{r \geqq R} g(r)=\inf \left[g(r): r \geqq R \text { and either } T r^{\alpha}=\|n\| /\|\alpha\| \text { or } r_{j}=R_{j} \text { for some } j\right] \text {. }
$$

But in case the set

$$
E=\left[r: r \in \mathscr{C}^{k+}, r \geqq R, \operatorname{Tr}^{\alpha}=\|n\| /\|\alpha\|\right]
$$

is not nul, the infimum of $g$ over $E$ is necessarily attained at a point $r$ of it with $r_{j}=R_{j}$ for some $j$. To see this let $n_{s} / \alpha_{s}=n / \alpha$ and let $H$ be the set of all positive integers $\leqq k$ and different from $s$. We observe that, for $r \in E$,

$$
g(r)=A \prod_{j \in H} r_{j}^{\left(n_{s} \alpha_{j} / \alpha_{s}-n_{j}\right)},
$$

where $A \in \mathscr{C}^{1+}$ and is independent of $r$. We are thus in a position to conclude that

$$
\begin{aligned}
\inf _{r \geqq R} g(r) & =\inf \left[g(r): r \geqq R, r_{j}=R_{j} \text { for some } j\right] \\
& =\min _{1 \leqq j \leqq k}\left[g(r): r \geqq R, r_{j}=R_{j}\right],
\end{aligned}
$$

which by our induction hypothesis implies (2.2)(a).

We now prove (b). It is easily seen, as in the case of the function $h$ in the proof of (a), that

$$
\inf \left[g(r): r_{j} \geqq R_{j}, r_{m}=R_{m} \text { for } 1 \leqq m \leqq k, m \neq j\right]=g(U) \text { or } g(R)
$$

according as $n_{j} / \alpha_{j} \geqq$ or $<T R^{\alpha}$, where $U=U(j) \in\left|\mathscr{C}^{k}\right|$ and is such that $T U^{\alpha}$ $=n_{j} / \alpha_{j}$ and $U_{m}=R_{m}$ for $1 \leqq m \leqq k, m \neq j$. By the hypothesis of (2.2)(b), the set

$$
J=\left[j: n_{j} / \alpha_{j} \geqq T R^{\alpha}\right]
$$


is not empty and hence by (a) it follows that

$$
\inf _{r \geqq R} g(r)=\min _{j \in J} g(U(j)) .
$$

But, for $j \in J, g(U) \geqq \inf \left[g(r): r \geqq R, T r^{\alpha}=n_{j} / \alpha_{j}\right]$, which again as in (a) may be shown to be $=g(S)$, where $S=S(j) \in \mathscr{C}^{k+}$ and is such that $S_{m}=R_{m}$ for $1 \leqq m \leqq k, \quad m \neq s \quad\left(n_{s} / \alpha_{s}\right.$ being $\left.=n / \alpha\right)$. Clearly $g(S(j)) \geqq g(U(s))$, so that $g(U(j)) \geqq g(U(s))$, for $1 \leqq j \leqq k$, which by (2.3) implies (b) and hence completes the proof of the lemma.

Our next lemma adds to the information provided by theorem 2.1 .

Lemma 2.4. Let $f$ denote the formal power series $\sum_{n \in I} a_{n} z^{n}$ in the indeterminates $z_{1}, \cdots, z_{k}$ over the field $\mathscr{C}^{1}$. Let $\rho \in\left|\mathscr{C}^{k}\right|$. Then

(a) $f$ defines an entire function over $\mathscr{C}^{k}$ admitting $\rho$ as a product order, iff to each $\alpha \in \mathscr{C}^{k+}$ and $\gg \rho$, but to no $\alpha \in \mathscr{C}^{k+}$ and $\ll \rho$ (when $\rho \gg \overline{0}$ ) correspond an $R=R(\alpha) \in \mathscr{C}^{k+}$ and a finite subset $J=J(\alpha) \subseteq I$ such that

$$
\left.\left|a_{n}\right| \leqq \inf _{r \geqq R}\left[1 / r^{n}\right) \exp r^{\alpha}\right], \quad \text { for } n \in I-J ;
$$

(b) part (a) holds with the last line of the same replaced by

$$
\left|a_{n}\right| \leqq\left[e R^{\alpha} /(n / \alpha)\right]^{n / \alpha} / R^{n}, \quad \text { for } n \in I-J
$$

(c) $f$ defines an entire function of finite product order and $\alpha \in \operatorname{int} P(f)$, iff $\alpha \in \mathscr{C}^{k+}$ and

$$
\limsup _{\|n\| \rightarrow+\infty}\{(n / \alpha) \ln \psi(n)\} /\left(-\ln \left|a_{n}\right|\right)<1 .
$$

Proof OF 2.4. In case $f$ defines an entire function and there exist $\alpha, R \in \mathscr{C}^{k+}$ such that $\ln ^{+} \mathscr{M}(r) \leqq r^{\alpha}$ for $r \geqq R$, we get, by Cauchy's Inequality (see [2]), that (2.5) holds with $J$ as the null set.

Let now $\alpha, R, \delta \in \mathscr{C}^{k+}$ and let $J$ be a finite subset of $I$ and let further (2.5) hold. To complete the proof of (a) at this stage we need only observe that $f$ defines an entire function and that

$$
\ln ^{+} \mathscr{M}(r) \leqq r^{\alpha+\delta}, \text { as } r \rightarrow+\bar{\infty} .
$$

But for any $r \geqq R$, the set $\left[\left|a_{n}\right| r^{n}: n \in I\right]$ is bounded so that the power series represented by $f$ converges in

$$
\left[z: z \in \mathscr{C}^{k},\left|z_{j}\right|<r_{j} \text { for } 1 \leqq j \leqq k\right] .
$$

Thus $f$ defines an entire function over $\mathscr{C}^{k}$. Further for $r \geqq R$ and $a \in \mathscr{C}^{1+}$ and $>1$,

$$
\sum_{n \in I-J}\left|a_{n}\right| r^{n} \leqq \sum_{n \in I-J}\left\{r^{n} /(a r)^{n}\right\} \exp (a r)^{\alpha} \leqq\{a /(a-1)\}^{k} \exp (a r)^{\alpha},
$$


so that

$$
\mathscr{M}(r) \leqq p(r) \exp (r)^{\alpha+\delta}, \quad \text { as } r \rightarrow+\bar{\infty},
$$

where $p(r) \rightarrow 0$ as $r \rightarrow+\bar{\infty}$, which implies what we are attempting to conclude. Hence follows (a).

(b) follows from (a) and lemma (2.2) (b).

We finally turn to (c). Let $f$ define an entire function of finite product order and let $\alpha \in$ int $P(f)$. There exists a positive real $t<1$ such that $t \alpha \in$ int $P(f)$. Hence there exists an $R \in \mathscr{C}^{k+}$ and a finite subset $J \subseteq I$ such that (2.6) holds with $t \alpha$ instead of $\alpha$. Now since $n /(t \alpha)=(n / \alpha) / t$ and $\left|a_{n}\right|^{1 /(n / \alpha)} \rightarrow 0$ as $\|n\| \rightarrow+\infty$ (see $\S 3$ of [2]), we get that

$$
-t \ln \left|a_{n}\right| \geqq(n / \alpha) \ln \psi(n)+\chi(n) \text {, as }\|n\| \rightarrow+\infty \text {, }
$$

where the function $\chi$ is such that $\chi(n) / \ln \left|a_{n}\right| \rightarrow 0$ as $\|n\| \rightarrow+\infty$. Thus our assumptions imply (2.7). To prove the other part of (c) we start with $\alpha \in \mathscr{C}^{k+}$ for which (2.7) holds. Hence there exists a positive real $t<1$ such that (2.8) holds where $\chi(n)=0$ as $\|n\| \rightarrow+\infty$, which implies that $\left|a_{n}\right|^{1 /(n / a)} \rightarrow 0$ as $\|n\| \rightarrow+\infty$ and the fact that (2.6) holds with $(t+1) \alpha / 2$ instead of $\alpha$, with any particular $R \in \mathscr{C}^{k+}$ and with $J=J(t, \alpha, R)$ as some finite subset of $I$. Hence by $(b) f$ defines an entire function in $\mathscr{C}^{k}$ and $\alpha \varepsilon$ int $P(f)$.

ProOF of 2.1. The theorem follows from lemma (2.4) (c) by noticing the fact that (2.7) is equivalent to the assertion that

$$
\limsup _{\|n\| \rightarrow+\infty}\left\{\left(n_{j} / \alpha_{j}\right) \ln \psi(n)\right\} /\left(-\ln \left|a_{n}\right|\right)<1, \text { for } 1 \leqq j \leqq k .
$$

To compare our results with the ones known in the case of the Fuks orders of $f$ (see theorem 26.2, $\$ 26$ of [2]) we state a trivial consequence of theorem (2.1) viz,

COROLlaRY 2.9. Let $\rho \in \mathscr{C}^{k+}$. Then $\rho$ is a product order of $f$, iff

$$
\limsup _{\|n\| \rightarrow+\infty}\{(n / \rho) \ln \psi(n)\} /\left(-\ln \left|a_{n}\right|\right)=1 .
$$

To complete a characterization of the product order set of an entire function over $\mathscr{C}^{k}$ (cf. theorem $26.3, \S 26$, of [2]) we present

THEOREM 2.10. Let $\rho \in\left|\mathscr{C}^{k}\right|$. Then there exists an entire function $f$ over $\mathscr{C}^{k}$ such that $P(f)=\left[\alpha: \alpha \in\left|\mathscr{C}^{k}\right|, \alpha \geqq \rho\right]$.

Proof OF 2.10. Let us first suppose that $\rho \gg \overline{0}$ and consider the power series defined by

$$
f(z)=\sum_{n I} a_{n} z^{n}, \text { for } z \in \mathscr{C}^{k}, \text { where } a_{n}=\psi^{-(n / n)}(n)
$$


We observe that $\{(n / \rho) \ln \psi(n)\} /\left(-\ln a_{n}\right)=1$ for $n \in I$ and that for any particular positive integer $j \leqq k, n / \rho=n_{j} / \rho_{j}$ for infinitely many $n \in I$ so that

$$
\limsup _{\|n\| \rightarrow+\infty}\left[n_{j} \ln \psi(n)\right] /\left(-\ln \left|a_{n}\right|\right)=\rho_{j}
$$

which by lemma (2.4) (c) and theorem (2.1) shows that $f$ defines an entire function of the required kind.

Let now $J=\left[j: \rho_{j}>0\right]$. In the general case we understand by $n / \rho$ the number $\max \left[n_{j} / \rho_{j}: j \in J\right]$, which we take as 0 if $J$ is null, and modify the definition of $f$ by taking the summation $\Sigma$ over

$$
\left[n: n \in I, n_{j}=0 \text { whenever } j \notin J\right]
$$

instead of over $I$ and observe that the $f$ has the required property.

The product type of $f$ corresponding to a product order $\in \mathscr{C}^{k+}$ does not seem to admit an elegant expression in terms of the Taylor coefficients of $f$. However the expression given below is an extension of the one well-known when $k=1$ and is good enough to serve our purpose (see theorem (2.16)).

THEOREM 2.11. Let $\rho \in \mathscr{C}^{k+}$ be a product order of $f$ and let $\tau$ (possibly $\left.+\infty\right)$ be the corresponding product type of the function. Then

$$
\tau=\limsup _{R \rightarrow+\infty} \limsup _{\|n\| \rightarrow+\infty}\left[(n / \rho)\left(\left|a_{n}\right| R^{n}\right)^{1 /(n / \rho)} /\left(e R^{\rho}\right)\right]
$$

and $\tau<+\infty$, iff $L<+\infty$.

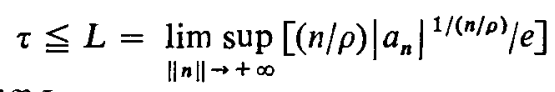

It is desirable to establish

Lemma 2.13. Let $f$ be the formal power series $\sum_{n \in I} a_{n} z^{n}$ in the indeterminates $z_{j}(1 \leqq j \leqq k)$ over the field $\mathscr{C}^{1}$. Let $\rho \in \mathscr{C}^{k+}$ and let $\tau \in\left|\mathscr{C}^{1}\right|$. Then $f$ defines an entire function over $\mathscr{C}^{k}$ and either $\rho$ is a product order and $\tau$ is the corresponding product type of $f$ or $\tau=0$ and $\rho \in \operatorname{int} P(f)$, iff (2.12) holds.

Proof of 2.13. Analogous to lemma (2.4) (a) we observe that $f$ defines and entire function over $\mathscr{C}^{k}$ and either $\rho$ is a product order and $\tau$ is the corresponding product type of $f$ or $\tau=0$ and $\rho \in$ int $P(f)$, iff to each $T \in \mathscr{C}^{1+}$ and $>\tau$, but to no $T \in \mathscr{C}^{1+}$ and $<\tau$ (when $\tau>0$ ) corresponds an $R \in \mathscr{C}^{k+}$ such that

$$
\left|a_{n}\right| \leqq \inf _{r \geqq R}\left[\left(1 / r^{n}\right) \exp \left(\operatorname{Tr}^{p}\right)\right] \text { as }\|n\| \rightarrow+\infty,
$$

which by lemma (2.2) (b) is equivalent to the fact that

$$
\left|a_{n}\right| \leqq\left[e T S^{\rho} /(n / \rho)\right]^{(n / \rho)} / S^{n} \text { as }\|n\| \rightarrow+\infty,
$$

for any $S \geqq R\left(S \in \mathscr{C}^{k+}\right)$ which implies the lemma. 
Proof of 2.11. Lemma (2.13) implies (a). (a) and the fact that

$$
n_{j} /(n / \rho)-\rho_{j} \leqq 0 \quad(1 \leqq j \leqq k)
$$

imply that $\tau \leqq L$ and that to each $T \in \mathscr{C}^{1+}$ and $>\tau$, when $\tau<+\infty$, corresponds an $R \in \mathscr{C}^{k+}$ such that $L \leqq T R^{\rho}$. We are thus in a position to conclude that (b) also holds.

REMARK 2.14. In the absence of a more elegant expression for $\tau$ in theorem (2.11) than the one provided by (2.12), it might be desirable to judge its nature through its relations with more elegant expressions like $L$. We however realise the falsehood of the statement (when $k>1$ ) that $\tau=L$ and even that of the statement that $\tau=0$ iff $L=0$, because of

EXAMPLE 2.15. Let $f$ be the function specified by

$$
f(z)=\sum_{1 \leqq j \leqq k} \exp z_{j},
$$

for $z \in \mathscr{C}^{k}(k>1)$. It is easily seen that $P(f)=\left[r: r \in \mathscr{C}^{k+}, r \geqq(1, \cdots, 1)\right]$ so that in particular $\rho=(1, \cdots, 1)$ is a product order for $f$. It is also easily seen that the product type of $f$ corresponding to $\rho$ is $\tau=0$, while the corresponding $L$ (of theorem (2.11)) is 1 .

We conclude this section with (cf. theorem $26.4, \S 26$ of [2]),

THEOREM 2.16. Let $\rho \in \mathscr{C}^{k+}$ and $\tau \in\left|\mathscr{C}^{1}\right|$ or $=+\infty$. Then there exists an entire function $f$ over $\mathscr{C}^{k}$ with $\rho$ as a product order and $\tau$ as the corresponding product type.

Proof of 2.16. Let us consider the three power series $\sum u_{n} z^{n}, \quad \sum v_{n} z^{n}$ and $\Sigma w_{n} z^{n}$ for $z \in \mathscr{C}^{k}$, where the summations $\Sigma$ are over $n \in I-(\overline{0})$ and

$$
\begin{aligned}
& u_{n}=(n / \rho)^{-(n / \rho)} . \\
& v_{n}=[(n / \rho) \ln (n / \rho)]^{-(n / \rho)}, \\
& w_{n}=[(n / \rho) / \ln (n / \rho)]^{-(n / \rho)} .
\end{aligned}
$$

To each $t \in \mathscr{C}^{1+}$, let us write $n(t)=\left(\left[\rho_{1} t\right], \cdots,\left[\rho_{k} t\right]\right)$, where $\left[\rho_{j} t\right]$ denotes the integer part of $\rho_{j} t$ and observe that

$$
n_{j}(t) /(n(t) / \rho) \rightarrow \rho_{j},
$$

as $t \rightarrow+\infty$, while in general $n_{j} /(n / \rho) \leqq \rho_{j}$ for $n \in I-\overline{0}(1 \leqq j \leqq k)$. Hence we are in a position to say that,

$$
\limsup _{\|n\| \rightarrow+\infty}\left[R^{n /(n / \rho)} / R^{\rho}\right]=1,
$$

for $R \in \mathscr{C}^{k+}$ and $\geqq(1, \cdots, 1)$. 
for $R \in \mathscr{C}^{k+}$ and $\geqq(1, \cdots, 1)$. (2.17) and lemma (2.13) together imply that the series $\Sigma u_{n} z^{n}$ defines an entire function $u$ over $\mathscr{C}^{k}$ with $\rho$ as a product order and $1 / e$ as the corresponding product type. Hence, when $0<\tau<+\infty$, the function $f$ defined by $f(z)=u\left(z(e \tau)^{1 /\|\rho\|}\right)$ for $z \in \mathscr{C}^{k}$, stands to prove the theorem. Using lemma (2.4) (c) (see also corollary (2.9)) we observe that the remaining two series respectively define entire functions, say $v$ and $w$, over $\mathscr{C}^{k}$, each with $\rho$ as a product order. Now by (2.17) and theorem (2.11) (a), it follows that $v$ and $w$ are respectively of product types 0 and $+\infty$ corresponding to $\rho$.

\section{Sum concepts and their relations with the product concepts}

Throughout this section $S(f)$ denotes the sum order set of $f$, while $P(f)$ and $\psi$ continue to have the same meanings as in the previous section. We start with

THEOREM 3.1. Let $\phi$ be a nonnegative real valued increasing function over $\left|\mathscr{C}^{k}\right|$.

(a) $\rho \in\left|\mathscr{C}^{k}\right|$ is a Fuks order of $\phi$, iff it is a sum order of $\phi$.

(b) Let $\rho \in \mathscr{C}^{k+}$ be a Fuks order and hence a sum order of $\phi$. Then $\tau \in\left|\mathscr{C}^{k}\right|$ is a Ronkin type of $\phi$ corresponding to $\rho$, iff it is a sum type of $\phi$ corresponding to $\rho$.

The proof of theorem 3.1 follows from the

Lemma 3.2. Let the function $h$ be defined by

$$
h(r)=\sum_{1 \leqq j \leqq k} T_{j} r_{j}^{j j}
$$

for $r \in\left|\mathscr{C}^{k}\right|$, where $T, \alpha \in \mathscr{C}^{k+}$. Then the following two propositions are equivalent:

(i) for any particular $\delta \in \mathscr{C}^{1+}, \phi(r) \leqq(1+\delta) h(r)$, as $r \rightarrow+\infty$;

(ii) for any particular $\delta \in \mathscr{C}^{1+}, \phi(r) \leqq(1+\delta) h(r)$, as $\|r\|=\Sigma r_{j} \rightarrow+\infty$.

Proof of 3.2. That (ii) implies (i) is obvious and we need only show that (i) implies (ii). Let (i) hold and let $\delta \in \mathscr{C}^{1+}$. By our assumption there exists an $R \in \mathscr{C}^{k+}$ such that

$$
\phi(s) \leqq(1+\delta / 2) h(s), \quad \text { for } \quad s \geqq R\left(s \in \mathscr{C}^{k+}\right) .
$$

Now let $\mathscr{J}$ be the class of all nonempty subsets of the set of positive integers $\leqq k$ and corresponding to any $s \in\left|\mathscr{C}^{k}\right|, J \in \mathscr{J}$, let $s(J) \in\left|\mathscr{C}^{k}\right|$ and be such that $s_{j}(J)$ $=s_{j}$ for $j \in J$ and $=0$ otherwise. To each $J \in \mathscr{J}$ corresponds an $S(J)$ which $\in \mathscr{C}^{1+}$, is $>\|R\|$ and is such that

$$
h(s) \leqq[(1+\delta) /(1+\delta / 2)] h(s(J)),
$$

whenever $s \in \mathscr{C}^{k+}, s_{j}=R_{j}$ for $j \notin J$ and $\|s\| \geqq S(J)$. Let us consider any $r \in\left|\mathscr{C}^{i}\right|$ such that

$$
\|r\| \geqq S=\max [S(J): J \in \mathscr{J}] \text {. }
$$


Let $s=\mathrm{s}(r, R) \in \mathscr{C}^{k+}$ be such that $s_{j}=\max \left(r_{j}, R_{j}\right)$, for $1 \leqq j \leqq k$. It follows that $\phi(r) \leqq \phi(s)$ which, since $s \geqq R$,

$$
\begin{aligned}
& \leqq(1+\delta / 2) h(s) \\
& \leqq(1+\delta) h(s(J)), \text { where } J=\left[j: r_{j}>R_{j}\right], \\
& \leqq(1+\delta) h(r)
\end{aligned}
$$

Theorem (3.1) practically takes away the need to discuss in detail the various properties of the sum concepts associated with $f$ in the presence of the literature on the Ronkin Fuks concepts (see $\$ 26.2$ of [2]). However it might be of interest to observe that through a development analogous to the one of our $\$ 2$ and through considerations as " $r \rightarrow+\infty$ " instead of " $\|r\| \rightarrow+\infty$ " one might reach deeper results with ease. We consider in particular (cf. theorem $26.2 \$ 26$ of [2]).

THEOREM 3.3. Let $f$ denote the formal power series $\sum a_{n} z^{n}$ over $\mathscr{C}^{1}$ in the indeterminates $z_{1}, \cdots, z_{k}$. Let $\rho, t \in \mathscr{C}^{k+}$.

(a) $f$ defines an entire function over $\mathscr{C}^{k}$ and $\alpha \in \operatorname{int} S(f)$, iff $\alpha \in \mathscr{C}^{k+}$ and

$$
\limsup _{\|n\| \rightarrow+\infty}\left[\left\{\left(\sum_{1 \leqq j \leqq k} n_{j} / \alpha_{j}\right) \ln \psi(n)\right\} /\left(-\ln a_{n} \mid\right)\right]<1
$$

(b) $f$ defines an entire function over $\mathscr{C}^{k}$ and either $\rho$ is a sum order for $f$ while $t$ belongs to the interior of the sum type set of $f$ corresponding to $\rho$ or $\rho \in \operatorname{int} S(f)$, iff

$$
\limsup _{\|n\| \rightarrow+\infty}\left[\left|a_{n}\right| \prod_{1 \leqq j \leqq k}\left\{n_{j} /\left(t_{j} e \rho_{j}\right)\right\}^{n_{j} / \rho_{j}}\right]^{1 / \Psi(n)}<1,
$$

where $\left\{n_{j} /\left(t_{j} e \rho_{j}\right)\right\}^{n_{j} / \rho_{j}}$ should be taken as 1 if $n_{j}=0$.

REMARK 3.6. We observe that (3.4) and (3.5) may be stated in the language of asymptotic bounds for $\left|a_{n}\right|$ as $\|n\| \rightarrow+\infty$ (cf. lemma (2.2) to (2.4) of [5]).

ProOF OF 3.3. The proof of (a) is similar to that of lemma (2.4) (c) and the proof of (b) employs the same techniques as the pfoor of lemma (2.13). The entire discussion of this theorem is primarily based on a more easily proved analogue of lemma (2.2) viz, that if $R, t, \alpha \in \mathscr{C}^{k+}$ and $n \in\left|\mathscr{C}^{k}\right|$ but $>\overline{0}$, then

$$
\inf _{r \geqq R}\left[\left(1 / r^{n}\right) \exp \left(\sum_{1 \leqq j \leqq k} t_{j} r_{j}^{\alpha j}\right)\right]=\prod_{1 \leqq j \leqq k} \inf _{r j \geqq R_{j}}\left[r_{j}^{-n_{j}} \exp \left(t_{j} r_{j}^{\alpha j}\right)\right]=\prod_{l \leqq j \leqq k} T_{j}
$$

where

$$
T_{j}= \begin{cases}\left(e t_{j} \alpha_{j} / n_{j}\right)^{n_{j} / \alpha_{j}} & \text { if } n_{j} / \alpha_{j} \geqq t_{j} R_{j}^{\alpha} \\ R_{j}^{-n j} \exp \left(t_{j} R_{j}^{\alpha j}\right) & \text { otherwise. }\end{cases}
$$


We next discuss the relations among the sum and product orders of $f$ in

THEOREM 3.7. Let $\mathscr{R}$ be the class of all sets of the form $\left[x: x \in\left|\mathscr{C}^{k}\right|, x \geqq \alpha\right.$ where $\alpha \in\left|\mathscr{C}^{k}\right|$. Let $P$ and $S$ be nonempty closed subsets of $\left|\mathscr{C}^{k}\right|$. Then $P$ and $S$ are respectively the product order set and the sum order set of an entire function fover $\mathscr{C}^{k}$, iff

(a) $P=\cap[E: E \in \mathscr{R}, E \supseteq S]$

(b) $S$ is octant shaped, i.e. $s \in S$ whenever $s \in\left|\mathscr{C}^{k}\right|$ and is $\geqq$ some $r \in S$, and is reciprocally convex, i.e., the set $\left[\alpha: \alpha \in \mathscr{C}^{k+},\left(1 / \alpha_{1}, \cdots, 1 / \alpha_{k}\right) \in\right.$ int $\left.S\right]$ is convex.

At this stage of the development the proof requires

Lemma 3.8. Let $\mathscr{R}$ be as in theorem (3.7). Then

$$
P(f)=\cap[E: E \in \mathscr{R}, E \supseteq S(f)]
$$

ProOF OF 3.8. Let us write

$$
Q=Q(f)=\cap[E: E \in \mathscr{R}, E \supseteq S(f)] .
$$

It is easy to see that int $S(f) \subseteq P(f)$ and hence that $S(f) \subseteq P(f)$. Hence by theorem (2.1) it follows that $Q \subseteq P(f)$.

Now let $\delta \in \operatorname{int} P(f)$, let

$$
\alpha \in F_{\delta}=\left[x: x \in \mathscr{C}^{k+}, \quad \Sigma\left(\delta_{j} / x_{j}\right) \leqq 1\right] .
$$

There exists a $p \in \mathscr{C}^{k+}$ such that $\|p\|=1$ and $\delta_{j} \leqq p_{j} \alpha_{j}$ for $1 \leqq j \leqq k$. For any $r \in \mathscr{C}^{k+}$ and $\geqq(1, \cdots, 1)$, we get that

$$
r^{\delta} \leqq \prod\left(r_{j}\right)^{\rho^{\alpha_{j}}} \leqq \Sigma p_{j} r_{j}^{\alpha j}, \text { when } \Pi=\prod_{1 \leqq j \leqq k},
$$

because of the fact that $\|p\|=1$ and because of the well-known relations between the weighed arithemetic and geometric means of the reals $r_{j}^{\alpha j}$ taken with weights $p_{j}$ $(1 \leqq j \leqq k)$. Thus we see that $F_{\delta} \subseteq S(f)$. Hence

$$
\left[x: x \in \mathscr{C}^{k+}, x \geqq \delta\right]=\cap\left[E: E \in \mathscr{R}, E \supseteq F_{\delta}\right] \subseteq Q,
$$

and this being true for every $\delta \in \operatorname{int} P(f)$ it follows that $P(f) \subseteq Q$. This together with the already proved fact that $Q \subseteq P(f)$ implies the lemma.

Proof of 3.7. If $S=S(f)$ and $P=P(f)$ for some entire function $f$, then (a) follows from lemma (3.8) while (b) follows from theorem (3.1) and the known properties of the Fuks order set of $f$ (see $\$ 26.2$ of [2]). Now let (a) and (b) hold. By theorem 26.3 of [2] and theorem (3.1) there exists an $f$ such that $S=S(f)$. Hence by lemma (3.8) $P=P(f)$ and this completes the proof.

Remark 3.9. Theorem 26.3 of [2] and lemma (3.8) imply theorem (2.10). 
THEOREM 3.10. (a) Let $S$ be a closed subset of $\left|\mathscr{C}^{k}\right|$ which is octant shaped and reciprocally convex (as explained in (3.7) (b)). Then $S$ is also convex.

(b) $S(f)$ is convex.

Proof OF 3.10. Let $\alpha, \beta \in S \cap \mathscr{C}^{k+}$ and let $\gamma=p \alpha+q \beta$ where $p, q \in \mathscr{C}^{1+}$ and $p+q=1$. The reciprocal convexity of $S$ implies that $\delta \in S$, where

$$
\delta_{j}=\left(p / \alpha_{j}+q / \beta_{j}\right)^{-1}
$$

for $1 \leqq j \leqq k$. But we notice that for any $1 \leqq j \leqq k$,

$$
\begin{gathered}
\left(\gamma_{j}-\delta_{j}\right)\left(p \beta_{j}+q \alpha_{j}\right)=\left(p^{2}+q^{2}\right) \alpha_{j} \beta_{j}+p q\left(\alpha_{j}^{2}+\beta_{j}^{2}\right)-\alpha_{j} \beta_{j} \geqq(p+q)^{2} \alpha_{j} \beta_{j}-\alpha_{j} \beta_{j} \\
=0,
\end{gathered}
$$

which shows that $\delta \leqq \gamma$. Since $S$ is octant-shaped it now follows that $\gamma \in S \cap \mathscr{C}^{k+}$, which shows that $S \cap \mathscr{C}^{k+}$ is convex. Hence follows (a). (b) is implied by (a) and theorem (3.7).

REMARK 3.11. The techniques of this paper are not powerful enough to decide whether certain properties of the product and sum concepts such as the reciprocal convexity of the sum order set would be true in the case of a more general real valued function than $\ln ^{+} \mathscr{M}$.

REMARK 3.12. The considerations "as $\|r\| \rightarrow+\infty$ " seem to require cautious handling at the outset, particularly in view of the perennial possibility " $0 \leqq r_{j}<1$ ". As an instance we consider (check around the relation (5.49), chapter V. of [2]),

EXAMPLE 3.13. Let $f$ be defined by

$$
f(z)=\exp \left[\frac{1}{2} z_{1}+\sum_{2 \leqq j \leqq k} z_{j}-(1 / 25)\right]
$$

Here $\ln \mathscr{M}(r)<\Sigma_{1 \leqq j \leqq k} r_{j}^{1}$ as $\|r\| \rightarrow+\infty$, but it is not true that

$$
\begin{gathered}
\ln \mathscr{M}(r)<r_{1}^{2}+\sum_{2 \leqq j \leqq k} r_{j}^{1} \\
\text { as }\|r\| \rightarrow+\infty
\end{gathered}
$$

as the relation fails to hold when $r_{1}=1 / 3$.

However if $\alpha \in A=\left[x: x \in \mathscr{C}^{k+}, \ln ^{+} \mathscr{M}(r)<\Sigma r_{j}^{\prime \prime}\right.$ as $\left.\|r\| \rightarrow+\infty\right]$, $\beta \in \mathscr{C}^{k+}$ and $\gg \alpha$, it is easy to see that $\beta$ also $\in A$.

\section{Gol'dberg concepts and their relations with the sum and product concepts}

Throughout this section $\mathscr{A}$ denotes the class of all bounded subsets $A$ of $\left|\mathscr{C}^{k}\right|$ which are open (w.r.t. $\left|\mathscr{C}^{k}\right|$ ), have nonempty intersection with $\mathscr{C}^{k+}$ and have the 
Abel property viz. that $r \in A$, whenever $r \in\left|\mathscr{C}^{k}\right|$ and is $\ll$ some $s \in A$. Throughout $\phi$ denotes a nonnegative real valued increasing function over $\left|\mathscr{C}^{k}\right|$ and

$$
M(R, \phi, A)=\sup \left[\phi(r): r \in\left|\mathscr{C}^{k}\right|, r \mid R \in A\right]
$$

for $R \in \mathscr{C}^{1+}$ and $A \in \mathscr{A}$. We refer to

$$
\rho=\rho(\phi, A)=\inf \left[\alpha: \alpha \in \mathscr{C}^{1+} \text { or } \alpha=+\infty, M(R, \phi, A) \leqq R^{\alpha} \text { as } R \rightarrow+\infty\left(\text { in }\left|\mathscr{C}^{1}\right|\right)\right]
$$

as the Goldberg order of $\phi$ w.r.t. $A$ and when $0<\rho<+\infty$ we refer to

$$
\begin{aligned}
\tau & =\tau(\phi, A)) \\
& =\inf \left[T: T \in \mathscr{C}^{1+} \text { or } T=+\infty, M(R, \phi, A) \leqq T R^{\rho} \text { as } R \rightarrow+\infty\left(\text { in }\left|\mathscr{C}^{1}\right|\right)\right]
\end{aligned}
$$

as the Gol'dberg type of $\phi$ w.r.t. $A$ and say that $\phi$ is of maximal, minimal, or mean Gol'dberg type (type-class) w.r.t. $A$ according as $\tau=+\infty, \tau=0$, or $0<\tau<+\infty$.

We attribute all the Gol'dberg concepts associated with $\ln ^{+} \mathscr{M}$ w.r.t. $A \in \mathscr{A}$ to $f$ w.r.t. $A$ or the set

$$
\left[z: z \in \mathscr{C}^{k},|z| \in A\right] .
$$

We first prove the preliminary result.

THEOREM 4.1. (a) the Gol'dberg order $\rho=\rho(\phi, A)$ of $\phi$ is independent of $A \in \mathscr{A}$.

(b) If $0<\rho<+\infty$, the Gol'dberg type-class of $\phi$ is independent of $A \in \mathscr{A}$.

(c) If $0<\rho<+\infty, \phi$ is of mean Gol'dberg type-class and $T \in \mathscr{C}^{1}$, then there exists an $A \in \mathscr{A}$ such that $\tau(\phi, A)=T$.

The proof essentially depends on the easily proved

LeMma 4.2. Let $A \in \mathscr{A}$. Let, for any $B \in \mathscr{A}, \mu \in \mathscr{C}^{1+}, \mu B$ denote the set $\left[x: x \in\left|\mathscr{C}^{k}\right|, x=\mu \alpha\right.$ for some $\left.\alpha \in B\right]$. Then

(a) $\rho(\phi, A)=\lim \sup _{R \rightarrow+\infty}\left[\left\{\ln ^{+} M(R, \phi, A)\right\} / \ln R\right]$;

(b) $\tau(\phi, A)=\lim \sup _{R \rightarrow+\infty}\left[M(R, \phi, A) / R^{\rho}\right]$, if $\rho=\rho(\phi, A) \in \mathscr{C}^{1+}$;

(c) for any $p \in \mathscr{C}^{1+}, \rho(\phi, p A)=\rho(\phi, A)$ and if this $\in \mathscr{C}^{1+}$, then $\tau(\phi, p A)$ $=p^{\rho} \tau(\phi, A)$, when $\rho=\rho(\phi, A)$.

ProOF OF 4.2. (a) and (b) admit direct proofs and we only need consider (c). Let $p \in \mathscr{C}^{1+}$. By (a)

$$
\begin{aligned}
\rho(\phi, A) & =\limsup _{R \rightarrow+\infty}\left[\left\{\ln ^{+} M(p R, \phi, A)\right\} / \ln (p R)\right] \\
& =\limsup _{\substack{R \rightarrow+\infty \\
\sin }}\left[\left\{\ln ^{+} M(R, \phi, p A)\right\} / \ln (p R)\right] \\
& =\rho(\phi, p A),
\end{aligned}
$$


which proves a part of (c) and brings out the basic ideas useful for proving the remainder of (c).

Proof of 4.1. Let $A, B \in \mathscr{A}$. There exist $p, q \in \mathscr{C}^{1+}$ such that $p B \subseteq A \subseteq q B$. Now by Lemma (4.2)

$$
\rho(\phi, B)=\rho(\phi, p B) \leqq \rho(\phi, A) \leqq \rho(\phi, q B)=\rho(\phi, B),
$$

which implies (a). The proof of (b) is similar. (c) is a trivial consequence of $(b)$ and (4.2) (c).

We now proceed to the central result of this section viz,

THEOREM 4.3. Let $P$ and $S$ be closed subsets of $\left|\mathscr{C}^{k}\right|$ and $\rho \in\left|\mathscr{C}^{1}\right|$ or $=+\infty$. Then $P, S$ and $\rho$ are respectively the product order set, the sum order set and the Gol'dberg order of an entire function over $\mathscr{C}^{k}$, iff either $P$ and $S$ are empty and $\rho=+\infty$ or $P$ and $S$ are nonempty, $\rho \in\left|\mathscr{C}^{1}\right|$ and (a) and (b) of theorem (3.7) hold along with the requirement that $(\rho, \cdots, \rho)$ of $\left|\mathscr{C}^{k}\right|$ is a boundary point of $S$.

The theorem easily follows from theorem (3.7) and the

Lemma 4.4. $\rho i$ the Gol'dberg order of $\phi$, iff either $(\rho, \cdots, \rho) \in\left|\mathscr{C}^{k}\right|$ and is a sum order of $\phi$ or $\rho=+\infty$ and $\phi$ is of infinite sum order.

ProOF OF 4.4. Let $p$ be the Gol'dberg order of $\phi$ and let $q$ be $+\infty$ or be such that $(q, \cdots, q)$ is a sum order of $\phi$ (there being one and only one such) according as $\phi$ is of infinite or finite sum order. Let $V$ be the unit cell $\left[x: x \in\left|\mathscr{C}^{k}\right|\right.$ and $\leqq(1, \cdots, 1)]$. The lemma is equivalent to the statement that $p=q$.

We first show that $p \geqq q$. This being obvious if $p=+\infty$, we shall suppose that $p<+\infty$. Let $\delta \in \mathscr{C}^{1+}$. Now there exists an $S \in \mathscr{C}^{1+}$ such that $M(R, \phi, V)$ $\leqq R^{p+\delta}$ for $R \geqq S\left(R \in \mathscr{C}^{1+}\right)$ and hence for $r \in\left|\mathscr{C}^{k}\right|$ such that $\|r\| \geqq k S$,

$$
\begin{aligned}
\phi(r) & \leqq \phi(R, \cdots, R)=M(R, \phi, V)=\max \left[\phi(r): r \in\left|\mathscr{C}^{k}\right|, r / R \in V\right] \leqq R^{p+\delta} \\
& \leqq \sum_{1 \leqq j \leqq k} r_{j}^{p+\delta},
\end{aligned}
$$

where $R=\max \left[r_{j}: 1 \leqq j \leqq k\right]$, which shows that $(p+\delta, \cdots, p+\delta) \in$ the sum order set of $\phi$, which, being true for any $\delta \in \mathscr{C}^{1+}$, implies that $p \geqq q$.

To show that $p \leqq q$ we need only observe that, for any $\delta \in \mathscr{C}^{1+}$

$$
M(R, \phi, V)=\phi(R, \cdots, R) \leqq k R^{q+\delta} \text {, as } R \rightarrow+\infty .
$$

Hence follows the lemma.

Further Theorem (4.3) enables us to take advantage of theorem (3.3) to give 
THEOREM 4.5. (a) Let $f$ denote, for the purpose of this theorem, the formal power series $\Sigma a_{n} z^{n}$ in the indeterminates $z_{1}, \cdots, z_{k}$ over the field $\mathscr{C}^{1}$. Let $\rho \in\left|\mathscr{C}^{1}\right|$. Then $f$ defines an entire function of Gol'dberg order $\rho$ over $\mathscr{C}^{k}$, iff

$$
\rho=\limsup _{\|n\| \rightarrow+\infty}\left[\{\|n\| \ln \psi(n)\} /\left(-\ln \left|a_{n}\right|\right)\right] .
$$

(b) If $f$ defines an entire function of Gol'dberg order $\rho$ over $\mathscr{C}^{k}$, then the last line of (a) holds (even if $\rho=+\infty$ ).

REMARK 4.6. This paper is essentially concerned with the "upper growth concepts" associated with the entire function $f$. The extensive literature in the case of one complex variable stimulates one to think about a number of other aspects associated with $f$ such as the "lower growth concepts", the concepts of "regular and other finer growths", their relevance and appropriateness in the context of the solution spaces of differential equations over $\mathscr{C}^{k}$ (as further indicated in [3] and other applications of theirs (cf. [5]). Once again the techniques of this paper are not powerful enough to handle even all the analogous issues that might be raised in the case of the "lower growth concepts", which (among others) are reserved to be pursued separately.

\section{Appendix}

We briefly consider a recorded attempt by Bose and Sharma (see (5.2), $\S 6$ and $\S 7$ of [1]) at introducing the order $\rho$ and type $\tau$ (when $0<\rho<+\infty$ ) of $f$ and obtaining their relations with the Taylor coefficients of $f$, when $k=2$, which we shall suppose hereafter. According to them,

$$
\rho=\inf \left[\alpha: \in \mathscr{C}^{1+} \text { or }=+\infty, \ln ^{+} \mathscr{M}(r) \leqq\left(r_{1} r_{2}\right)^{\alpha} \text { as } r \rightarrow+\infty\right]
$$

and when $0<\rho<+\infty$,

$$
\begin{aligned}
& \tau=\inf \left[T: T \in \mathscr{C}^{1+} \text { or }=+\infty, \ln ^{+} \mathscr{M}(r) \leqq T\left(r_{1}^{\rho}+r_{2}^{\rho}\right)\left(\text { and not } T\left(r_{1} r_{2}\right)^{\rho}\right)\right. \text { as } \\
& r \rightarrow+\bar{\infty}]
\end{aligned}
$$

They, in particular, arrive at some expressions for $\rho$ and $\tau$ in terms of the lim sup's of certain functions formed from $a_{m}$, as $m \rightarrow+\bar{\infty}$ (and not as $\|m\| \rightarrow+\infty$ ) (see theorems IV, V of [1]).

Apart from the questions of interest (in the $\tau$ in particular) and of the soundness of reasoning, the conclusions (with " $m \rightarrow+\infty$ ") of Bose and Sharma, under consideration, are basically invorrect, for the simple reason that one might easily give counter examples by considering entire functions over $\mathscr{C}^{2}$ which are "essentially bad functions over $\mathscr{C}^{1}$ ', For example the function $f$ defined by $f(z)=\exp \exp z$ for $z \in \mathscr{C}^{2}$, contradicts their theorem IV. 


\section{References}

[1] S. K. Bose, and D. Sharma, 'Integral functions of two complex variables', Compositio Mathematica, 15 (1963), 210-226.

[2] B. A. Fuks, Introduction to the theory of analytic functions of several complex variables (Amer. Math. Soc., (1963)).

[3] J. Gopala Krishna, 'Maximum term of a power series in one and several complex variables', Pacific Jour. Math. 29 (1969), 609-622.

[4] J. Gopala Krishna, 'Probabilistic techniques leading to a Valinon-type theorem in several complex variables', Ann. Math. Stat., 41 (1970), 2126-2129.

[5] Fred Gross, 'Generalized Taylor series and orders and types of entire functions of several complex variables', Trans. Amer. Math. Soc. 120 (1965), 124-144.

Department of Mathematics

University of Illinois

Urbana, lllinois, U. S. A.

Department of Mathematics

Andhra University

Waltair, A. P., India 